\title{
Optimization of Catheter Based rtPA Thrombolysis in a Novel In Vitro Clot Model for Intracerebral Hemorrhage
}

\author{
Naureen Keric, ${ }^{1}$ Julia Masomi-Bornwasser, ${ }^{1}$ Hendrik Müller-Werkmeister, ${ }^{1}$ \\ Sven Rainer Kantelhardt, ${ }^{1}$ Jochem König, ${ }^{2}$ Oliver Kempski, ${ }^{3}$ and Alf Giese ${ }^{1,4}$ \\ ${ }^{1}$ Department of Neurosurgery, University Medical Center, Johannes Gutenberg University, Mainz, Germany \\ ${ }^{2}$ Institute of Medical Biostatistics, Epidemiology and Informatics (IMBEI), University Medical Center, Johannes Gutenberg University, \\ Mainz, Germany \\ ${ }^{3}$ Institute for Neurosurgical Pathophysiology, University Medical Center, Johannes Gutenberg University, Mainz, Germany \\ ${ }^{4}$ OrthoCentrum Hamburg, Hansastr. 1-3, Hamburg, Germany
}

Correspondence should be addressed to Naureen Keric; naureen.keric@unimedizin-mainz.de

Received 26 November 2016; Accepted 7 March 2017; Published 26 March 2017

Academic Editor: Serge Marbacher

Copyright (C) 2017 Naureen Keric et al. This is an open access article distributed under the Creative Commons Attribution License, which permits unrestricted use, distribution, and reproduction in any medium, provided the original work is properly cited.

\begin{abstract}
Hematoma lysis with recombinant tissue plasminogen activator (rtPA) has emerged as an alternative therapy for spontaneous intracerebral hemorrhage (ICH). Optimal dose and schedule are still unclear. The aim of this study was to create a reliable in vitro blood clot model for investigation of optimal drug dose and timing. An in vitro clot model was established, using $25 \mathrm{~mL}$ and $50 \mathrm{~mL}$ of human blood. Catheters were placed into the clots and three groups, using intraclot application of rtPA, placebo, and catheter alone, were analyzed. Dose-response relationship, repetition, and duration of rtPA treatment and its effectiveness in aged clots were investigated. A significant relative end weight difference was found in rtPA treated clots compared to catheter alone $(p=0.002)$ and placebo treated clots $(p<0.001)$. Dose-response analysis revealed $95 \%$ effective dose around $1 \mathrm{mg}$ rtPA in 25 and $50 \mathrm{~mL}$ clots. Approximately $80 \%$ of relative clot lysis could be achieved after $15 \mathrm{~min}$ incubation. Lysis of aged clots was less effective. A new clot model for in vitro investigation was established. Our data suggest that current protocols for rtPA based ICH therapy may be optimized by using less rtPA at shorter incubation times.
\end{abstract}

\section{Introduction}

Spontaneous intracerebral hemorrhage occurs in $10-15 \%$ of all stroke patients and is still a major cause of strokerelated death and disability [1-3]. Optimal therapy is still controversial. Prospective studies and randomized trials have shown equally poor outcome for best medical treatment or open surgery for evacuation of the hematoma, whereas one study suggests that the latter might be beneficial for selected patients with lobar hematomas not deeper than $1 \mathrm{~cm}$ from brain surface and a GCS between 9 and 12 [4-8].

In consideration of these results the interest in minimal invasive procedures has grown. Stereotactic frame-based or image-guided frame-less catheter placement and rtPA lysis of ICH have shown to be safe and effective in volume reduction [9-12]. In the past 2 decades this therapy has emerged to a well-established fast and easy procedure in many neurosurgical units [13]. However rtPA dosing mainly has been based on clinical experience. No published data are available from (i) in vitro or in vivo studies systematically analyzing optimal timing of rtPA administration, (ii) optimal dosing of the drug, and (iii) duration of efficient treatment. Not surprisingly some authors reported adverse events, which might be dose dependent, such as occurrence of an rtPA induced delayed cytotoxic perifocal edema. This was observed in animal models and clinical settings [14-17], while some other studies did not confirm these findings [18, 19]. A trial on minimal invasive $\mathrm{ICH}$ lysis using an intralesional catheter is presently conducted [20].

In order to establish optimal dose and timing for rtPA therapy, however high numbers of repetitive investigations are necessary to achieve valid and significant results. Studies 


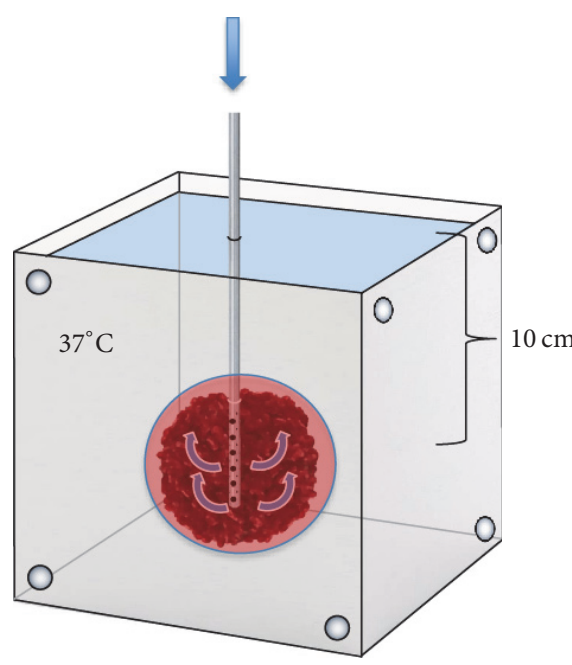

(a)

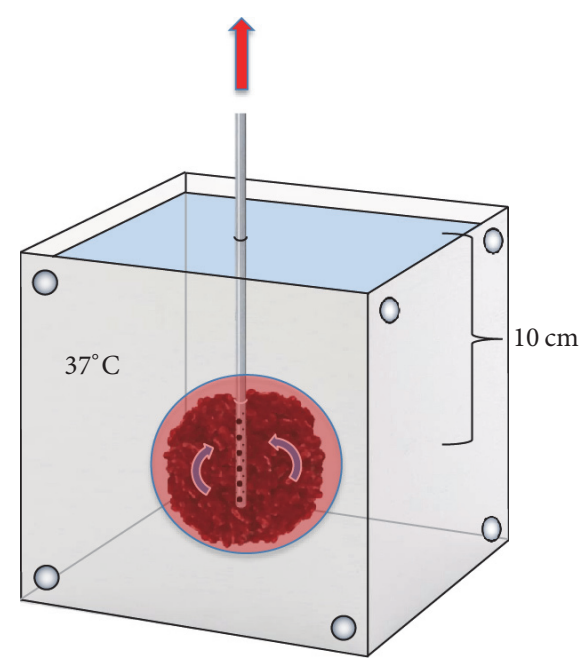

(b)

Figure 1: Blood clots submerged $10 \mathrm{~cm}$ in a $37^{\circ} \mathrm{C}$ water bath. An EVD is placed in all clots for drug administration (a). After treatment the liquefied fraction is drained by a gravity based drainage system (b).

in large ICH animal models are very expensive. An obvious alternative is an in vitro ICH model, which allows a high number of repetitions under controlled conditions. We here present an easy and robust in vitro ICH model in which we investigated optimal timing and dosing of rtPA lysis [21, 22].

\section{Material and Methods}

2.1. Blood Clot Preparation. We collected blood from the cubital vein of healthy volunteers into $20 \mathrm{~mL}$ syringes (BD Discardit, Germany). In vitro blood clots were produced from $25 \mathrm{~mL}$ or $50 \mathrm{~mL}$ of human blood supplemented with $10 \mathrm{IE}$ of thrombin (bovine plasma thrombin, Sigma, Germany, final concentration $10 \mathrm{IE} / 500 \mu \mathrm{L}$ ) in a balloon tightly closed and incubated $1.5 \mathrm{~h}$ in an incubator at $37^{\circ} \mathrm{C}$ (Heraeus Instruments, Germany). The application of thrombin has been adapted from an intravascular clot model $[23,24]$. Before treatment clots were weighed, the clot and serum fraction were separated carefully by a fine mesh and weighed individually and afterwards placed back into the balloon for treatment. After clot production the clots were randomized to the different treatment groups.

2.2. In Vitro ICH Model. An external ventricular drain (EVD) (Neuromedex ${ }^{\circledR} \mathrm{GmbH}$, Switzerland, 9 F, $30 \mathrm{~cm}$ length, 20 holes with $1 \mathrm{~mm}$ diameter) catheter was placed into all clots, mimicking the intracranial situation of a lysis catheter, and connected to a gravity based EVD drainage system (Neuromedex ${ }^{\circledR} \mathrm{GmbH}$, Switzerland) and placed $10 \mathrm{~cm}$ below the blood clot level. The clots were placed $10 \mathrm{~cm}$ below surface in a water bath at $37^{\circ} \mathrm{C}$. Temperature was constantly monitored by a thermometer ( $\mathrm{PH}$ Meter, WTW $\mathrm{GmbH}$, Germany) (Figure 1).

After randomization and corresponding to the different experimental protocols the EVD system was opened and the liquefied fraction of the hematoma was drained by gravity.
After treatment the remainder of each clot was weighed to assess the relative weight reduction of the clot.

2.3. Spontaneous Thrombolysis, Carrier Effect, and rtPA Lysis. In the first setting we investigated the amount of spontaneous lysis, a potential carrier effect, and the rtPA lysis effect. Group 1 ( $n=6,25 \mathrm{~mL}$ clots) was treated with an EVD to drain the liquid fraction after $1 \mathrm{~h}$ incubation in a $37^{\circ} \mathrm{C}$ water bath to quantify the spontaneous lysis process. In group 2 ( $n=6$, $25 \mathrm{~mL}$ clots) $5 \mathrm{~mL}$ of $0,9 \% \mathrm{NaCl}$ was administered to the clots. Corresponding to group 1 the drain was opened after $1 \mathrm{~h}$. Three clots $(50 \mathrm{~mL})$ were treated with a dose of $3 \mathrm{mg}$ rtPA diluted in a volume of $5 \mathrm{~mL}$ in group 3. Drains were opened after $1 \mathrm{~h}$ incubation. Total volume of carrier $(\mathrm{NaCl})$ or rtPA was $5 \mathrm{~mL}$ to exclude possible effects of different carrier volumes. Following this, relative clot weight reduction was compared for all groups.

2.4. Dose-Response Relationship. A dose-response relationship was evaluated in five groups each consisting of three $25 \mathrm{~mL}$ blood clots with five different doses of rtPA $(0.5 ; 0.9$; $1.2 ; 2 ; 3 \mathrm{mg}$, treatment time $60 \mathrm{~min}$ ) using the clot model. Clots were weighed before and after treatment. Similar to this, 5 different doses of rtPA were applied in $50 \mathrm{~mL}$ clots $(0.5 ; 0.9$; $1.2 ; 2 ; 3 ; \mathrm{mg}$, treatment time $60 \mathrm{~min})$. Furthermore each rtPA treated clot was compared to a placebo $(5 \mathrm{~mL} 0.9 \% \mathrm{NaCl})$ treated clot of the same blood donor. The differences of weight of the treated and the control blood clots were statistically analyzed to assess the lysis effect of each rtPA dose.

2.5. Optimal Treatment Time. In order to investigate the optimal treatment time for $\mathrm{rtPA}, 25 \mathrm{~mL}$ clots were treated with an optimized rtPA dose of $1 \mathrm{mg}$ by different periods of time. After rtPA administration, the EVD system was opened after 5, 15, 30, and $60 \mathrm{~min}$ (each time point in replicates 
of 3 clots). Relative weight reduction after treatment was compared.

2.6. Effectiveness of rtPA in Different Old Clots. Clots of different ages were produced as described above $(1.5 \mathrm{~h}, 24 \mathrm{~h}$, and $48 \mathrm{~h}$; each group consisting of $n=3$ ). One mg rtPA was applied repetitively four times. During each treatment rtPA was applied and remained in the clot for $15 \mathrm{~min}$; then liquid fraction was drained for $10 \mathrm{~min}$. Clots were weighed before and after treatment.

2.7. Statistical Analysis. We summarized results by reporting mean \pm standard deviation. For comparison of spontaneous thrombolysis, carrier effect and rtPA lysis, and effectiveness of $1 \mathrm{mg}$ rtPA in different aged clots statistical analysis was performed by one-way analysis of variance. $95 \%$ confidence intervals for all parameters were reported. Two-sided $p$ values below 0.05 were considered as statistically significant. Analysis was performed with SigmaPlot 12.0. (Systat Software, Inc., USA) and GraphPad Prism (version 6.0).

For statistical analysis of the dose-response relationship of rtPA, we fitted a three-parameter logistic model and estimated the $50 \%$ and $95 \%$ effective dose with $95 \%$ confidence intervals based on the fitted model [25].

$$
f(x,(b, d, e))=\frac{d}{1+\exp \{b(\log (x)-\log (e))\}} .
$$

Analysis was performed with R software, version 3.0.1: R Core Team (2013), R: A language and environment for statistical computing (R Foundation for Statistical Computing, Vienna, Austria, URL http://www.R-project.org/). For model fitting the $\mathrm{R}$ package DRC was used [25].

\section{Results}

3.1. Reliability of the Clot Model. A total number of 44 clots of human blood were created. The solid clot and liquid serum parts were separated and weighed. The solid part had an average weight of $21.76 \pm 1.03 \mathrm{~g}$ and the average liquid serum fraction was $3.81 \pm 0.88 \mathrm{~g}$. The low variance in weight shows the consistency of clot formation in this in vitro ICH model (Figures 2(a) and 2(b)) [21, 22].

3.2. Spontaneous Thrombolysis, Carrier Effect, and RtPA Lysis Effect. The control group (drain only) showed a mean relative end weight of $64.96 \pm 5.26 \%$ of initial weight. The drain plus carrier treated clots with a mean relative end weight of $69.44 \pm 6.67 \%$ showed no significant difference compared to the control group $(p=0.222)$. The $\mathrm{rtPA}(3 \mathrm{mg})$ treated clots with a relative end weight of $46.01 \pm 6.1 \%$ showed a significant difference compared to control $(p=0.002)$ and to drain plus carrier alone treated clots $(p<0.001)$ (Figure 3 ) $[21,22]$.

3.3. Dose-Response Relationship of rtPA in $25 \mathrm{~mL}$ Clots. A dose-response relationship was evaluated in five groups each consisting of three $25 \mathrm{~mL}$ blood clots using five different doses of $\operatorname{rtPA}(0.5 ; 0.9 ; 1.2 ; 2 ; 3 \mathrm{mg})$ and compared to a placebo $(5 \mathrm{~mL} 0,9 \% \mathrm{NaCl})$ treated clot from the same donor. Clots

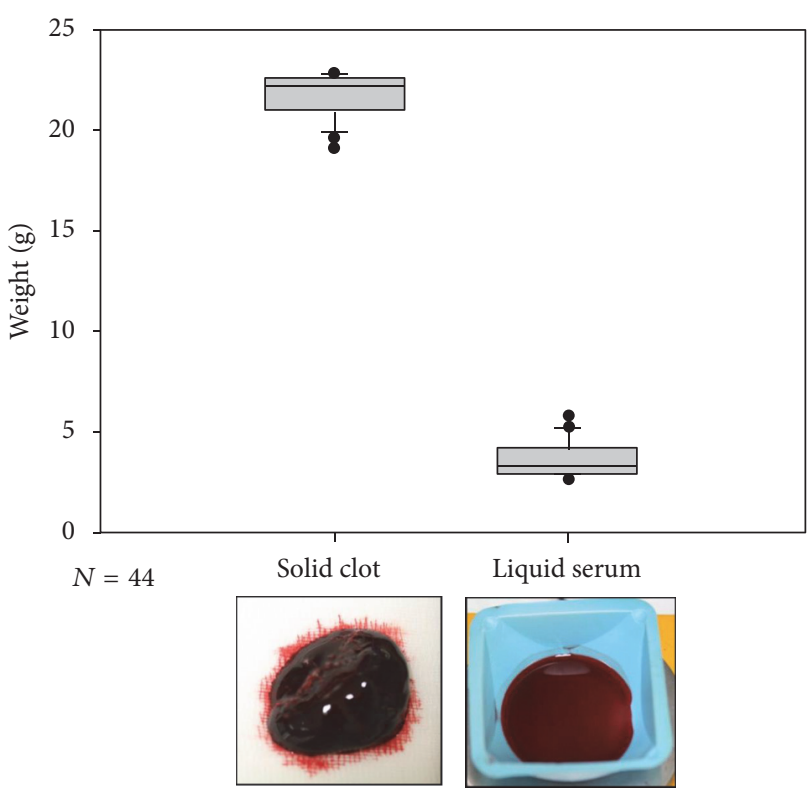

(a)

(b)

Figure 2: Clot formation in vitro; $n=44$. Weight of clot $(21.76$ $\pm 1.03 \mathrm{~g})$ and serum $(3.81 \pm 0.88 \mathrm{~g})$ part is illustrated in box plots showing the low mean variance. (a) Showing the solid part of the blood clot and (b) the liquid serum part of the clot.

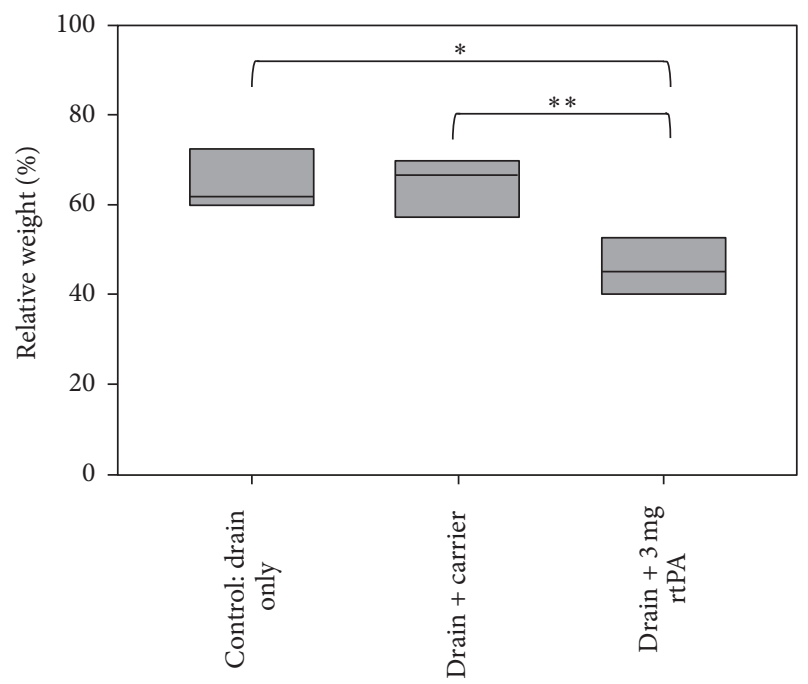

FIGURE 3: Comparison of spontaneous thrombolysis, carrier effect, and rtPA lysis as box plots $(n=6)$. The control group: blood clots treated with drain only had a relative weight of $64.96 \pm 5.26 \%$. Blood clots treated with $5 \mathrm{~mL}$ of carrier solution: $0.9 \% \mathrm{NaCl}$ had a relative weight after treatment of $69.44 \pm 6.67 \%$. Clots treated with $3 \mathrm{mg}$ of rtPA showed a relative weight of $46.01 \pm 6.1 \%$. ${ }^{* *} p<0.001$; $^{*} p=$ 0.002 .

were weighed before and after treatment. The rtPA treated group showed a relative posttreatment weight of $60.03 \pm 1.76 \%$ when treated with $0.5 \mathrm{mg}$ rtPA, $49.06 \pm 0.98 \%$ when treated with $0.9 \mathrm{mg} \mathrm{rtPA}, 48.78 \pm 2.14 \%$ when treated with $1.2 \mathrm{mg}$ $\mathrm{rtPA}, 46.34 \pm 4.68 \%$ when treated with $2 \mathrm{mg} \mathrm{rtPA}$, and $46.01 \pm$ $6.1 \%$ when treated with $3 \mathrm{mg}$ rtPA. The control group had a 


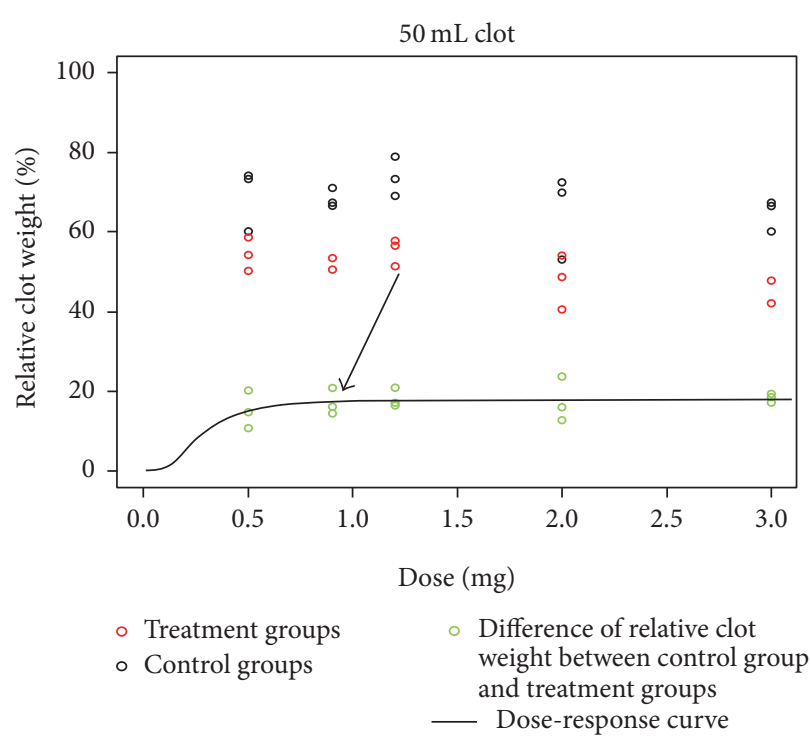

(a)

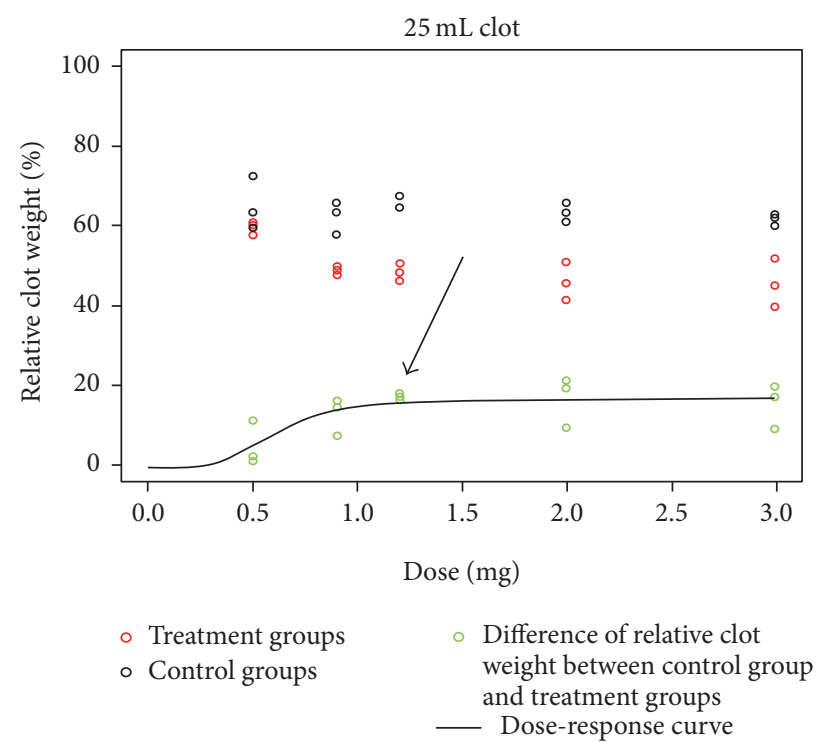

(b)

Figure 4: (a) A dose-response relationship was evaluated in five groups each consisting of three $25 \mathrm{~mL}$ blood clots with five different doses of rtPA $(0.5 ; 0.9 ; 1.2 ; 2 ; 3 \mathrm{mg})$ : treatment group (red) and control group (black). The relative posttreatment clot weight is shown on the $y$-axis. The differences of weight of the treated and the control blood clots showed the effect of lysis of each rtPA dose (green). The black line shows the dose-response relationship of rtPA. The arrow indicates the 95\% effective dose of $1.2 \pm 0.52 \mathrm{mg}$ rtPA. (b) Similar to (a) a dose-response relationship was evaluated for $50 \mathrm{~mL}$ blood clots in five groups each consisting of three $50 \mathrm{~mL}$ blood clots with five different doses of rtPA $(0.5 ; 0.9 ; 1.2 ; 2 ; 3 \mathrm{mg})$ : treatment group (red) and control group (black). The relative posttreatment clot weight is shown on the $y$-axis. The differences of weight of the treated and the control blood clots showed the effect of lysis of each rtPA dose (green). The black line shows the dose-response relationship of rtPA. The arrow indicates the $95 \%$ effective rtPA dose of $0.84 \pm 1.07 \mathrm{mg}$ rtPA.

mean relative posttreatment weight of $64.04 \pm 3.67 \%$. The $95 \%$ effective dose (ED95) was $1.2 \pm 0.52 \mathrm{mg} \mathrm{rtPA}$; the $50 \%$ effective dose (ED50) was $0.6 \pm 0.1 \mathrm{mg}$ (Figure $4(\mathrm{a}))[21,22]$.

3.4. Dose-Response Relationship of rtPA in $50 \mathrm{~mL}$ Clots. The same experiment was performed in $50 \mathrm{~mL}$ clots. The treatment group showed a relative posttreatment weight of $54.23 \pm 4.41 \%$ treated with $0.5 \mathrm{mg}$ rtPA, $51.41 \pm 1.79 \%$ treated with $0.9 \mathrm{mg} \mathrm{rtPA}, 55.23 \pm 3.36 \%$ treated with $1.2 \mathrm{mg} \mathrm{rtPA}$, $47.91 \pm 6.96 \%$ treated with $2 \mathrm{mg} \mathrm{rtPA}$, and $45.98 \pm 3.06 \%$ treated with $3 \mathrm{mg}$ rtPA. The control group had a mean relative posttreatment weight of $68.94 \pm 6.52 \%$. The $95 \%$ effective dose (ED95) was $0.84 \pm 1.07 \mathrm{mg}$ rtPA; the $50 \%$ effective dose (ED50) was $0.28 \pm 0.35 \mathrm{mg}$ (Figure 4(b)) [21, 22].

3.5. Optimal Treatment Time and Lysis Rate. Assuming a maximum lysis rate of $100 \%$ after $1 \mathrm{~h}$, lysis rates after different exposure times to rtPA were analyzed in $25 \mathrm{~mL}$ clots. The normalized rate of lysis after $5 \mathrm{~min}$ exposure to $\mathrm{rtPA}$ was $53.22 \pm 3.9 \%$; after $15 \mathrm{~min}$ it was $79.41 \pm 1.7 \%$ and after $30 \mathrm{~min}$ $85.38 \pm 1.5 \%$ (Figure 5) [21, 22].

3.6. Effectiveness of rtPA in Clots of Different Age. Fibrinolytic treatment with rtPA is less effective in aged clots. There was a significant weight difference of 90 min old clots compared to $24(p<0.0001)$ and $48 \mathrm{~h}$ old clots $(p=0.0002)$ during the first treatment (Table 1). During the second treatment

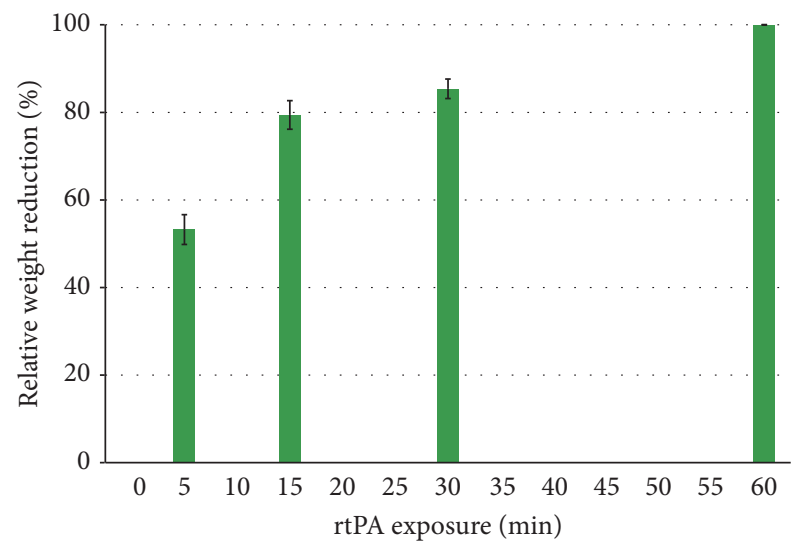

FIGURE 5: The graph shows normalized relative end weights after different time exposures of the blood clots to rtPA for $5,15,30$, and $60 \mathrm{~min}$ determining that $100 \%$ lysis takes place within $60 \mathrm{~min}$. Error bars represent standard error per condition; values are rescaled for $100 \%$ per $60 \mathrm{~min}$; app. $80 \%$ lysis happens during $15 \mathrm{~min}$. Each bar represents $n=3$ clots.

there was still a significant difference between the $90 \mathrm{~min}$ aged clots compared to $24 \mathrm{~h}$ aged clots $(p=0.0059)$ (Table 1 ). Repetitive rtPA treatment showed decreasing effectiveness in weight reduction. The bulk weight reduction was achieved by the first two treatments (Figure 6) [21, 22]. 
TABLE 1: Relative weight after repetitive treatment with $1 \mathrm{mg}$ rtPA in different aged clots: $90 \mathrm{~min}, 24 \mathrm{~h}$, and $48 \mathrm{~h}$.

\begin{tabular}{|c|c|c|c|c|}
\hline $\begin{array}{l}\text { Treatment } \\
\text { cycles }\end{array}$ & $90 \mathrm{~min}$ & $24 \mathrm{~h}$ & $48 \mathrm{~h}$ & Statistics \\
\hline 1 & $55.23 \pm 7.13 \%$ & $77.78 \pm 8.2 \%$ & $75.71 \pm 2.35 \%$ & $\begin{array}{l}90 \text { min versus } 24 \mathrm{~h}: p<0.0001 \\
90 \text { min versus } 48 \mathrm{~h}: p=0.0002\end{array}$ \\
\hline 2 & $44.23 \pm 5.04 \%$ & $59.02 \pm 4.78 \%$ & $54.83 \pm 7.83 \%$ & 90 min versus $24 \mathrm{~h}: p=0.0059$ \\
\hline 3 & $41.62 \pm 3.11 \%$ & $49.89 \pm 5.65 \%$ & $48.07 \pm 5.17 \%$ & \\
\hline 4 & $34.28 \pm 2.33 \%$ & $44.69 \pm 9.77 \%$ & $44.54 \pm 5.86 \%$ & \\
\hline
\end{tabular}

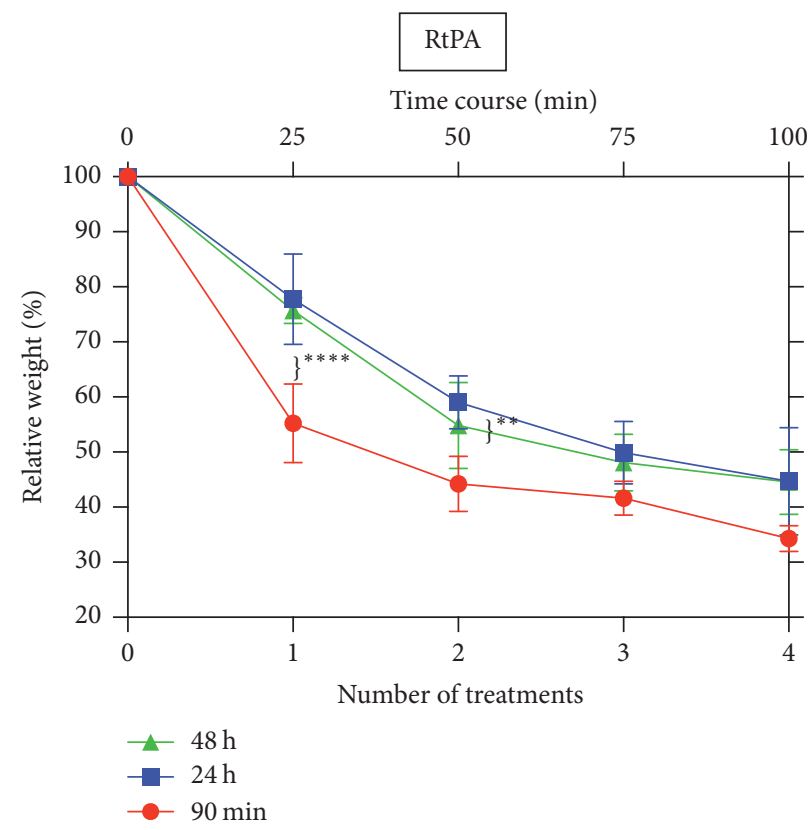

Figure 6: Effectiveness of $1 \mathrm{mg}$ rtPA in different old clots: $90 \mathrm{~min}$ (red), $24 \mathrm{~h}$ (blue), and $48 \mathrm{~h}$ (green): the graph shows the relative weight in percent ( $y$-axis) after 1 till 4 treatment cycles ( $x$-axis) $(n=3)$. On top axis the time course shows cycles of 25 min, consisting of 15 min of rtPA-exposure and 10 min drainage period. $* * * *$ indicates the significant weight difference of 90 min old clots compared to $24 \mathrm{~h}(p<0.0001)$ and $48 \mathrm{~h}$ old clots $(p=0.0002)$ during the first treatment. $* *$ indicates the significant weight difference of the 90 min old clots compared to 24 h old clots $(p=0.0059)$ during the second treatment.

\section{Discussion}

In the present study to the best of our knowledge we investigated for the first time a systematic analysis of fibrinolytic therapy of rtPA (supplemental flowchart illustrates the experimental work; see Supplementary Material available online at https://doi.org/10.1155/2017/5472936). We established a novel in vitro ICH clot model $[21,22]$. 44 blood clots were produced from 25 or $50 \mathrm{~mL}$ of human blood from healthy volunteers. The supplementary use of thrombin according to published methods of in vitro micro intravascular clots stabilized the clot independent from donor $[23,24]$. The model proved to be highly reproducible in terms of clot formation of solid hematomas and serum fraction (Figure 2) [21, 22]. This setting allowed an easy workflow and a high number of repetitions under controlled conditions and avoided the need of a large ICH animal model [26].

In the first experimental series we evaluated the effect of rtPA lysis in comparison to control groups. Our findings indicate that normal saline irrigation of clots has no significant fibrinolytic effect $[21,22]$. The rtPA treated clots in contrast had a significant loss of their relative end weight, confirming that fibrinolysis takes place in this experimental in vitro setting (Figure 3 ). These results demonstrating an app. 55\% volume reduction after a single dose of $3 \mathrm{mg} \mathrm{rtPA}$ are in line with several experimental and clinical studies investigating the fibrinolytic potential of rtPA in ICH [9$19,26-28]$. In one of the first clinical series reported already 2 decades ago Lippitz et al. reported on fibrinolytic therapy after initial stereotactic aspiration of the hematoma. In 10 patients the authors yielded about $60 \%$ volume reduction by aspiration and a total of $84 \%$ hematoma removal after additional repetitive $\mathrm{rtPA}$ administration of $3 \mathrm{mg}$ daily over 1-3 days [9]. The latest published study, an analysis of a Phase II trial patient collective for minimal invasive surgery with rtPA, showed that this therapy is well tolerated and effective. The authors even found a decrease of perihematomal edema 
in the rtPA and aspiration treated group compared to the aspiration only treated group. They assume no neurotoxic rtPA effects on perifocal brain tissue when it is administered into the clot. Moreover the larger hematoma volume and reduction of toxic metabolites in the aspiration and rtPA group may lead to a decreased perifocal edema [19]. Until now no clear correlation of rtPA doses and the occurrence or size of a perifocal edema has been published. Interestingly the animal and clinical studies reporting of rtPA related perifocal edema applied relative high cumulative doses of $\mathrm{rtPA}[14,16$, 17, 26]. The ongoing phase III MISTIE trial will reveal possible dose-related side effects.

To further characterize the optimal dose of rtPA in different clot sizes, we performed a dose-response analysis in $25 \mathrm{~mL}$ and $50 \mathrm{~mL}$ blood clots. The $95 \%$ effective dose of rtPA in $25 \mathrm{~mL}$ clots was $1.2 \pm 0.52 \mathrm{mg}$ and $0.84 \pm 1.07 \mathrm{mg}$ in $50 \mathrm{~mL}$ clots $[21,22]$. We interpreted the lower dose in larger clot as not significant. In the Phase II MISTIE trial the rtPA dose was evaluated in 2 arms, while arm 1 received $0.3 \mathrm{mg}$ every 8 hours, up to 9 times; arm 2 received $1 \mathrm{mg}$ every 8 hours and up to 9 times in 72 hours. The investigators chose these dose regimens with reference to the recommendation of the American Heart Association in the application of intravenous rtPA and by the experiences of different clinical centers [9-14]. Some authors applied $1 \mathrm{mg}$ rtPA per $10 \mathrm{~mL}$ hematoma, whereas hematoma volume was assessed by the formula $A \times B \times C / 2$. The total rtPA doses ranged in these series depending on hematoma volume from 5 to $16 \mathrm{mg}[10$, $14,15]$. In our in vitro study surprisingly a larger clot did not require a higher rtPA dose. This might result from the relative hematoma surface, which can be reached by rtPA molecules per administration via the EVD. Possibly the catheter perforations and design play a role in this phenomenon. However the relative activity of $\mathrm{rtPA}$ in $1 \mathrm{mg} \mathrm{rtPA}$ seems to be sufficient or excessive even for larger hematoma volumes like $50 \mathrm{~mL}[21,22]$. The effect of repetition and timing remain unclear.

After assessment of an optimal rtPA dose we investigated the optimal exposure time of the clot to rtPA. In the published clinical series already mentioned above and in the protocol of the phase III MISTIE trial the drain was closed for 1 hour after rtPA application. Then the drain was opened for passive flow by gravity $[9-11,14,15,18-20]$. Considering this clinical practice we assumed that the fibrinolytic effect, which can be reached by a single rtPA dose in 1 hour, was determined as $100 \%$. Assuming this, in our in vitro series the lysis rates after $5 \mathrm{~min}$ exposure to rtPA were $53.22 \pm 3.9 \%$, after $15 \mathrm{~min}$ $79.41 \pm 1.7 \%$, and after $30 \mathrm{~min} 85.38 \pm 1.5 \%$ [21, 22]. These results correspond well to the half-life of rtPA, which is about $6 \mathrm{~min}$ [29]. But it raises the question, whether it is necessary to close drains for 1 hour, if app. $80 \%$ of the lysis can be achieved within $15 \mathrm{~min}[21,22]$. Translating these findings to the clinical situation with a faster opening of the drain could lower time of increased intracranial pressure and increase the effectiveness of hematoma evacuation.

The question of rtPA efficacy in older hematomas is still a matter of debate. The phase III MISTIE protocol excludes patients with symptoms more than $24 \mathrm{~h}$ prior to the initial diagnostic CT scan and surgery should be intended in 72 hours after ictus [20]. In our in vitro series we found a significant higher lysis rate in newly formed clots of $1.5 \mathrm{~h}$ compared to the $24 \mathrm{~h}$ and $48 \mathrm{~h}$ old clots [21, 22]. There was no difference in the lysis rate of $24 \mathrm{~h}$ and 48 old clots, suggesting that the relevant changes causing rtPA resistance take place within the first $24 \mathrm{~h}$. Furthermore, repetitive rtPA administrations did not result in a linear decrease of clot volume. The largest volume reduction occurred after the first 2 rtPA applications.

\section{Limitation and Advantages of This Model}

These results, however, have to be interpreted with caution. This in vitro model does not consider the perifocal environment of the brain tissue surrounding the intracerebral hemorrhage. Many inhibiting and activating factors may influence the maturation of clots but also the activity of the administered drugs. Large animal models may be superior to our in vitro model in assessing this question.

The advantages of this model are its reproducibility and reliability of clot size and structure and the usefulness in numerous future experiments, focusing not only on rtPA kinetics. Effects and kinetics of several other lytic drugs and their combination may be rapidly compared and investigated in this model. Furthermore the lytic activity of different ultrasound modes and physics can be assessed easily in this in vitro model. The model offers the perspective of assessing an individualized fibrinolytic therapy using lytic drugs alone, sonothrombolysis alone, or a combination of lytic drugs and sonothrombolysis. Therapeutic issues concerning clot age and coagulation status are important, which can be easily screened in such a model system before testing in an animal model and finally in the patient.

\section{Conclusion}

We established an easy and robust in vitro model of $\mathrm{ICH}$, which allows a high number of repetitive experiments under controlled conditions. We applied this model to assess the optimal dose and timing of rtPA lysis in human blood clots and found a surprisingly low optimal dose of only $1 \mathrm{mg}$ rtPA independent of the clot size $(25,50 \mathrm{~mL}$, resp.). Further we showed that $80 \%$ of the lysis occurs within the first $15 \mathrm{~min}$ of incubation. The data suggests that current protocols for rtPA based ICH therapy could possibly be optimized by using smaller doses and shorter incubation times. This might in the future allow a faster reduction of intracranial pressure than achieved in current clinical protocols.

\section{Ethical Approval}

This study was approved by the local Ethical Committee of Rhineland palatinate.

\section{Consent}

All blood samples were taken after informed consent of healthy voluntaries. 


\section{Disclosure}

Results of this article are parts of the ongoing doctoral thesis of Hendrik Müller-Werkmeister.

\section{Conflicts of Interest}

The authors declare that there are no conflicts of interest regarding the publication of this paper.

\section{Authors' Contributions}

Naureen Keric and Julia Masomi-Bornwasser equally contributed to this work.

\section{Acknowledgments}

The authors thank all the voluntary blood donors, who made this study possible.

\section{References}

[1] N. Kreitzer and O. Adeoye, "An update on surgical and medical management strategies for intracerebral hemorrhage," Seminars in Neurology, vol. 33, no. 5, pp. 462-467, 2013.

[2] V. L. Roger, A. S. Go, D. M. Lloyd-Jones et al., "Heart disease and stroke statistics-2012 update: a report from the American Heart Association," Circulation, vol. 125, no. 1, pp. e2-e220, 2012.

[3] L. B. Morgenstern, J. C. Hemphill III, C. Anderson et al., "Guidelines for the management of spontaneous intracerebral hemorrhage: a guideline for healthcare professionals from the American Heart Association/American Stroke Association," Stroke, vol. 41, no. 9, pp. 2108-2129, 2010.

[4] E. M. Manno, J. L. D. Atkinson, J. R. Fulgham, and E. F. M. Wijdicks, "Emerging medical and surgical management strategies in the evaluation and treatment of intracerebral hemorrhage," Mayo Clinic Proceedings, vol. 80, no. 3, pp. 420433, 2005.

[5] H.-G. Hårdemark, N. Wesslén, and L. Persson, "Influence of clinical factors, CT findings and early management on outcome in supratentorial intracerebral hemorrhage," Cerebrovascular Diseases, vol. 9, no. 1, pp. 10-21, 1999.

[6] A. D. Mendelow, B. A. Gregson, H. M. Fernandes et al., "Early surgery versus initial conservative treatment in patients with spontaneous supratentorial intracerebral haematomas in the International Surgical Trial in Intracerebral Haemorrhage (STICH): a randomised trial," The Lancet, vol. 365, no. 9457, pp. 387-397, 2005.

[7] A. D. Mendelow, B. A. Gregson, E. N. Rowan, G. D. Murray, A. Gholkar, and P. M. Mitchell, "Early surgery versus initial conservative treatment in patients with spontaneous supratentorial lobar intracerebral haematomas (STICH II): a randomised trial," The Lancet, vol. 382, no. 9890, pp. 397-408, 2013.

[8] B. A. Gregson, J. P. Broderick, L. M. Auer et al., "Individual patient data subgroup meta-analysis of surgery for spontaneous supratentorial intracerebral hemorrhage," Stroke, vol. 43, no. 6, pp. 1496-1504, 2012.

[9] B. E. Lippitz, L. Mayfrank, U. Spetzger, J. P. Warnke, H. Bertalanffy, and J. M. Gilsbach, "Lysis of basal ganglia haematoma with recombinant tissue plasminogen activator (rtPA) after stereotactic aspiration: initial results," Acta Neurochirurgica, vol, 127, no. 3-4, pp. 157-160, 1994.

[10] C. Schaller, V. Rohde, B. Meyer, and W. Hassler, "Stereotactic puncture and lysis of spontaneous intracerebral hemorrhage using recombinant tissue-plasminogen activator," Neurosurgery, vol. 36, no. 2, pp. 328-335, 1995.

[11] T. Morgan, M. Zuccarello, R. Narayan, P. Keyl, K. Lane, and D. Hanley, "Preliminary findings of the minimally-invasive surgery plus rtPA for intracerebral hemorrhage evacuation (MISTIE) clinical trial," Acta Neurochirurgica, Supplementum, no. 105, pp. 147-151, 2008.

[12] D. W. Newell, M. M. Shah, R. Wilcox et al., "Minimally invasive evacuation of spontaneous intracerebral hemorrhage using sonothrombolysis," Journal of Neurosurgery, vol. 115, no. 3, pp. 592-601, 2011.

[13] E. Abdu, D. F. Hanley, and D. W. Newell, "Minimally invasive treatment for intracerebral hemorrhage," Neurosurgical Focus, vol. 32, no. 4, article E3, 2012.

[14] V. Rohde, I. Rohde, R. Thiex et al., "Fibrinolysis therapy achieved with tissue plasminogen activator and aspiration of the liquefied clot after experimental intracerebral hemorrhage: rapid reduction in hematoma volume but intensification of delayed edema formation," Journal of Neurosurgery, vol. 97, no. 4, pp. 954-962, 2002.

[15] R. Thiex, V. Rohde, I. Rohde et al., "Frame-based and frameless stereotactic hematoma puncture and subsequent fibrinolytic therapy for the treatment of spontaneous intracerebral hemorrhage," Journal of Neurology, vol. 251, no. 12, pp. 1443-1450, 2004.

[16] R. Thiex and S. E. Tsirka, "Brain edema after intracerebral hemorrhage: mechanisms, treatment options, management strategies, and operative indications," Neurosurgical Focus, vol. 22, no. 5, article E6, 2007.

[17] N. Keric, G. S. Maier, U. Samadani et al., “Tissue plasminogen activator induced delayed edema in experimental porcine intracranial hemorrhage: reduction with plasminogen activator inhibitor-1 administration," Translational Stroke Research, vol. 3, supplement 1, pp. 88-93, 2012.

[18] J. R. Carhuapoma, R. J. Barrett, P. M. Keyl, D. F. Hanley, and R. R. Johnson, "Stereotactic aspiration-thrombolysis of intracerebral hemorrhage and its impact on perihematoma brain edema," Neurocritical Care, vol. 8, no. 3, pp. 322-329, 2008.

[19] W. A. Mould, J. R. Carhuapoma, J. Muschelli et al., "Minimally invasive surgery plus recombinant tissue-type plasminogen activator for intracerebral hemorrhage evacuation decreases perihematomal edema," Stroke, vol. 44, no. 3, pp. 627-634, 2013.

[20] D. Hanley, "Minimally Invasive Surgery plus Rt-PA for ICH Evacuation Phase III (MISTIE III)," 2013, http://www.clinicaltrials.gov.

[21] H. Mueller-Werkmeister, Optimierung der katheterbasierten rtPA-Fibrinolyse in einem in vitro Modell der intrazerebralen Blutung [Ph.D. thesis], Johannes Gutenberg University of Mainz, Mainz, Germany.

[22] J. Masomi, N. Keric, H. Mueller-Werkmeister, O. Kempski, and A. Giese, "Intralesional rtPA and ultrasound for in vitro thrombolysis of experimental intracerebral hemorrhage," in Proceedings of the DGNC Annual Meeting, Karlsruhe, Germany, June 2015.

[23] C. W. Francis, P. T. Onundarson, E. L. Carstensen et al., "Enhancement of fibrinolysis in vitro by ultrasound," The Journal of Clinical Investigation, vol. 90, no. 5, pp. 2063-2068, 1992. 
[24] C. Wright, K. Hynynen, and D. Goertz, "In vitro and in vivo high-intensity focused ultrasound thrombolysis," Investigative Radiology, vol. 47, no. 4, pp. 217-225, 2012.

[25] C. Ritz and J. C. Streibig, “Bioassay analysis using R,” Journal of Statistical Software, vol. 12, article 5, 2005.

[26] K. R. Wagner, G. Xi, Y. Hua et al., "Lobar intracerebral hemorrhage model in pigs: rapid edema development in perihematomal white matter," Stroke, vol. 27, no. 3, pp. 490-497, 1996.

[27] K. R. Wagner, G. Xi, Y. Hua et al., "Ultra-early clot aspiration after lysis with tissue plasminogen activator in a porcine model of intracerebral hemorrhage: edema reduction and blood-brain barrier protection," Journal of Neurosurgery, vol. 90, no. 3, pp. 491-498, 1999.

[28] R. J. Barrett, R. Hussain, W. M. Coplin et al., "Frameless stereotactic aspiration and thrombolysis of spontaneous intracerebral hemorrhage," Neurocritical Care, vol. 3, no. 3, pp. 237-245, 2005.

[29] T. Karow and R. Lang-Roth, "Fibrinolytika," in Allgemeine und Spezielle Pharmakologie und Toxikologie, p. 172, Thomas Karow, Köln, Germany, 2009. 


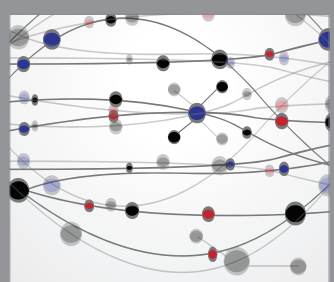

The Scientific World Journal
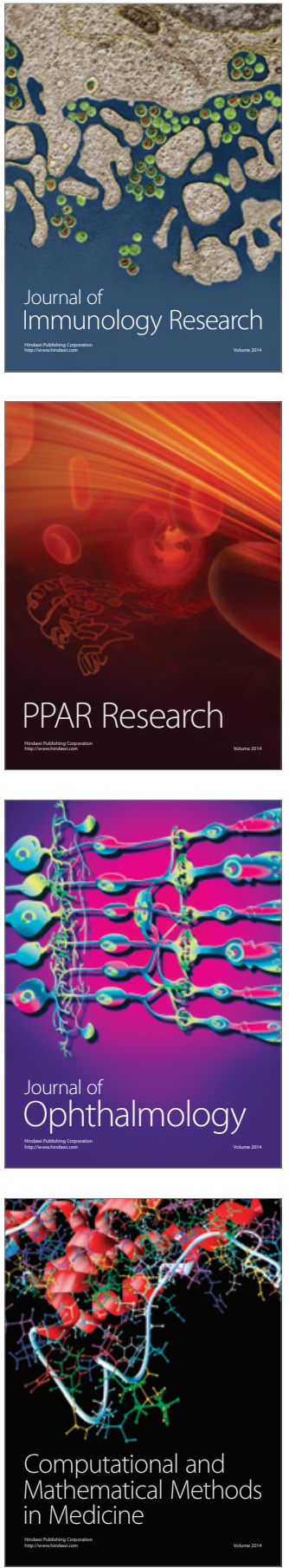

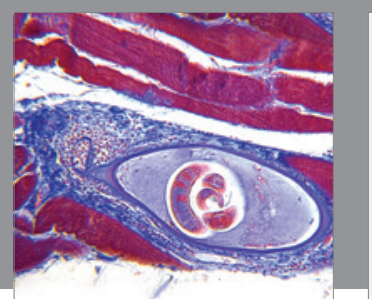

Gastroenterology Research and Practice
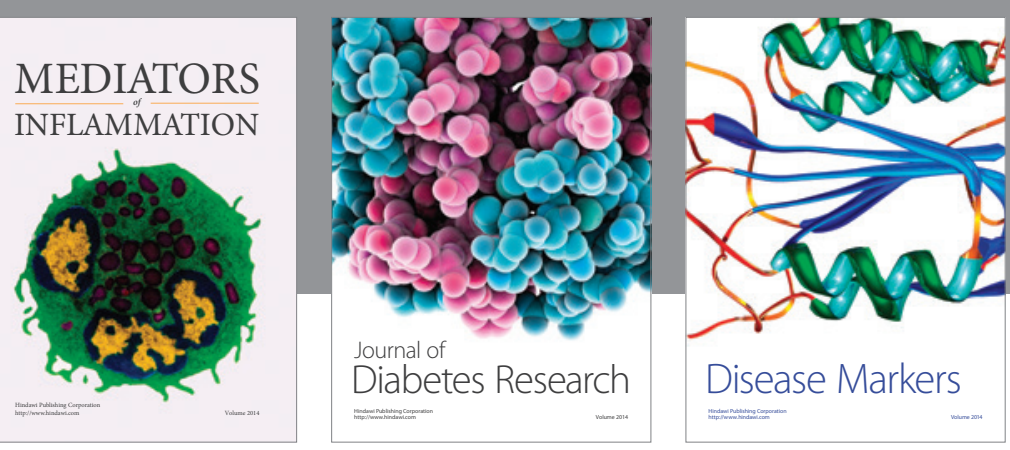

Disease Markers

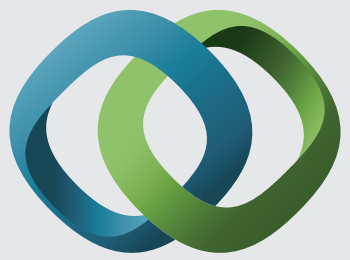

\section{Hindawi}

Submit your manuscripts at

https://www.hindawi.com
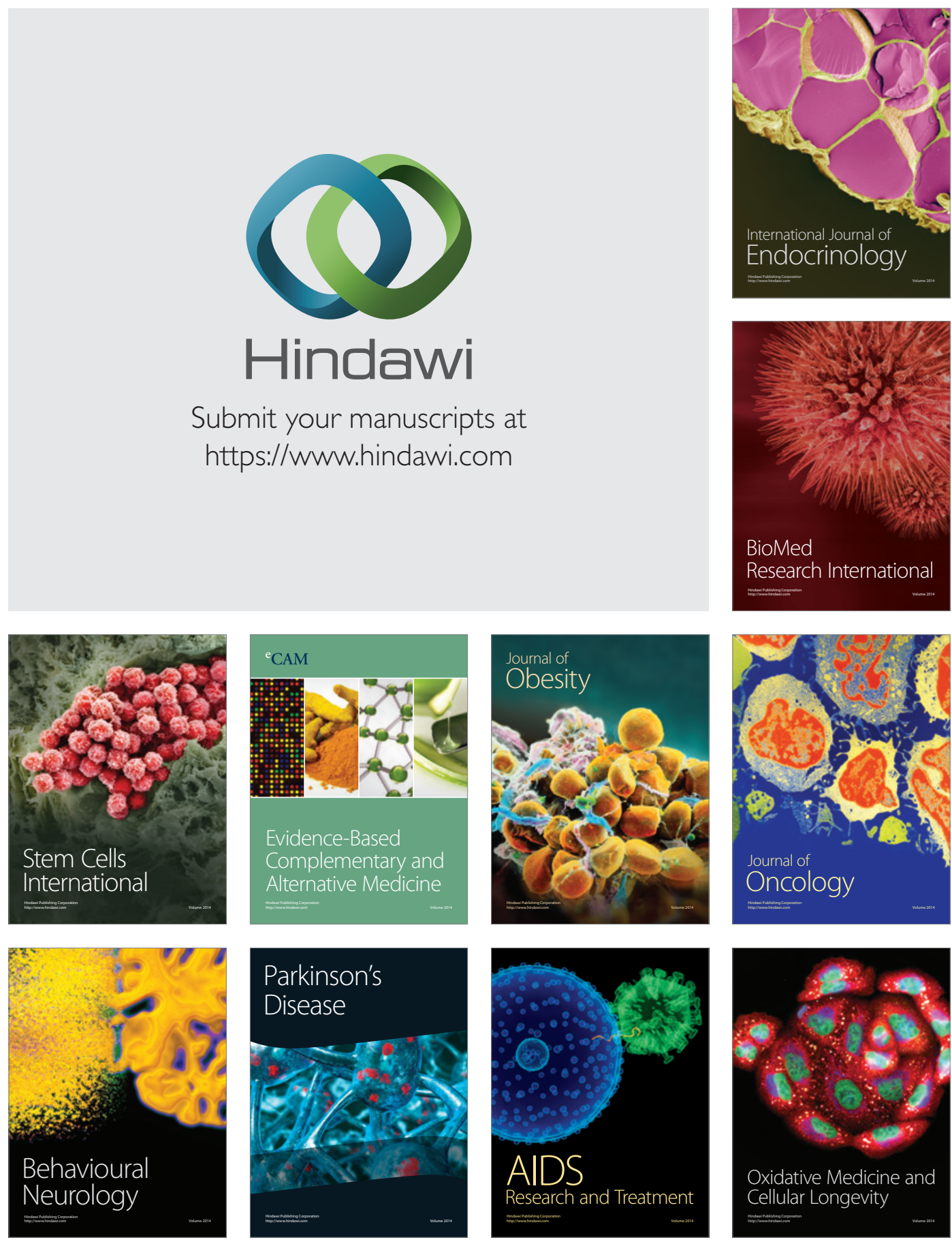